\title{
Un "tuiterazo" por la independencia: una experiencia del uso de Twitter para la enseñanza de Historia
}

\section{A hashtag movement for independence: using Twitter to teach history}

\author{
María Mercedes Zerega-Garaycoa ${ }^{1}$ \\ ${ }^{1}$ Universidad Casa Grande - UCG, Guayaquil, Equador \\ Correo a/Mail to: María Mercedes Zerega-Garaycoa tzerega@casagrande.edu.ec
}

Financiamiento/Funding: Universidad Casa Grande; Liceo Los Andes

Recibido/Submitted: 21 Oct. 2015; Aceptado/Approved: 09 Nov. 2015

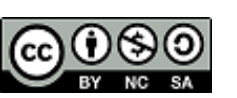

\begin{abstract}
Copyright (c) 2015 Zerega-Garaycoa, M. M.. Todo el contenido de la revista (incluyendo instrucciones, política editorial y modelos) está bajo una licencia Creative Commons Atribución-NoComercial-Compartirlgual 3.0 No Adaptada. Siendo publicados por esta revista, artículos son de libre uso en ambientes educativos, de investigación y no comerciales, con atribución de autoría obligatoria. Más información en http://ojs.c3sl.ufpr.br/ojs2/index.php/atoz/about/submissions\#copyrightNotice.
\end{abstract}

\begin{abstract}
Resumen
Introducción: Es necesario que los estudiantes dejen de comprender un evento histórico de forma unidimensional y se aproximen a él desde los relatos de sus actores. ¿Cómo lograr que lo hagan desarrollando empatía? ¿Se puede usar las tecnologías del presente para "jugar" al pasado? Este estudio resume un proyecto de aula que organizó un juego de rol a través de Twitter denominado "tuiterazo por la independencia" en la materia de Historia tomando como marco la Enseñanza para la Comprensión (EpC). El objetivo era comprender las posiciones de actores de la independencia latinoamericana y desarrollar empatía.

Método: Se evaluaron el nivel de comprensión y la percepción de los estudiantes sobre el desarrollo de empatía promovido por la actividad, así como su satisfacción con ésta a partir de un cuestionario constituido de preguntas abiertas para evaluar aprendizajes y preguntas cerradas y escalas para medir su empatía y nivel de satisfacción. El cuestionario fue aplicado a 72 jóvenes estudiantes de bachillerato.

Resultados: Los estudiantes entendieron que para comprender un evento histórico debe comprenderse el punto de vista diferentes actores y alcanzaron buenos niveles de comprensión del papel de cada actor en la independencia. Además indicaron que contribuyó en buena medida a desarrollar su empatía y tuvo un alto nivel de satisfacción.

Conclusiones: La red social Twitter puede ser utilizada para el desarrollo de juego de roles de carácter histórico y reforzar las comprensiones de la historia como un conjunto de relatos producidos por actores que tienen diferentes roles y posiciones alrededor de éste.
\end{abstract}

Palabras clave: Enseñanza de Historia; Twitter; Redes sociales; Juego de roles

\begin{abstract}
Introduction: There are difficulties in understanding historical time. It is necessary that students stop seeing historic events in a one-dimensional way and see them by means of the actor's tale instead. How can teachers achieve that students do so by developing empathy? Can one use current technology in order to "play" the past? This research summarizes a class project that organized a game using role-play via Twitter, called "Twitting for independence" in a course in History taking Teaching for Understanding (TfU) as reference framework. The objective was to comprehend the position of the actors of Latin-American independence and build empathy regarding their roles.

Method: It was evaluated the level of perception and comprehension of the students concerning the development of empathy promoted by this activity, as well as their satisfaction with this, applying a open questionnaire in order to measure their learning, as well as closed questions and scales to measure their empathy and satisfaction level. The questionnaire was applied to 72 young high school students.

Results: Students learnt that in order to understand a historic event it had to be seen from the actors' different points of view. They reached good levels of understanding of the role each actor played in achieving Latin American Independence and indicated that the activity largely contributed to developing their empathy.

Conclusions: The social network Twitter can be used to develop historical character role-plays and reinforce the comprehension of History as an ensemble of tales produced by actors with different roles and positions around it.
\end{abstract}

Keywords: History teaching; Twitter; Social Networks; Role-Play

\section{INTRODUCCIÓN}

Este estudio describe una práctica pedagógica que usó Twitter para la enseñanza de Historia en el Liceo Los Andes, un colegio privado de la ciudad de Guayaquil. Su modelo educativo asume el constructivismo y otros enfoques como la Enseñanza para la Comprensión (EpC). En Sociales se pretende que el estudiante de bachillerato se desplace de la noción de "historia", a la concepción de "historias" en plural y pueda realizar lecturas de los eventos desde diferentes puntos de vista considerando actores en diversas posiciones sociales y de poder ${ }^{1}$.

El programa de Historia de Bachillerato 1 se centra en América Latina. Entre sus módulos se encuentra el tema de la independencia que tiene como objetivo central el análisis crítico de la independencia latinoamericana,

\footnotetext{
${ }^{1}$ El programa de Sociales se rige por el Ministerio de Educación, que especifica entre sus objetivos relacionados al tiempo histórico: la comprensión de categorías relacionadas al tiempo, la noción de antes-después, la construcción de explicaciones históricas multicausales, la imaginación del pasado y el juicio de los personajes a partir de sus contextos, entre otros
} 
considerando causas y actores principales involucrados (españoles, pensadores latinoamericanos, criollos e indígenas). Se innovó la enseñanza del módulo con la realización de un juego de roles en la red social Twitter.

Desde el punto de vista tecnológico, la institución oferta en su currículo la materia de computación. En el 2015 la Dirección Académica define como objetivo la incorporación de la tecnología de forma integral en secundaria. El programa implica el diseño de desempeños a realizarse en conjunto entre computación y cada materia y la incorporación de plataformas educativas y colaborativas para el aprendizaje.

A partir del juego de rol, el estudio se pregunta por el impacto de éste en la comprensión de la posición de distintos actores de la independencia, así como el nivel de comprensión alcanzado sobre el papel de cada uno de éstos y el desarrollo de la empatía y la satisfacción producida por la actividad.

Las redes sociales se están incorporando a la educación. Se mencionan algunas aplicaciones educativas del microblogging, entre las que se encuentran el debate de temas de interés, la escritura colaborativa, la interacción con docentes, el manejo de proyectos, la reflexión, entre otras (De Haro, 2010; Grosseck \& Holotescu, 2008). Existen algunos autores que plantean el uso de la red social Twitter para fines educativos, pero casi todas esas experiencias pedagógicas la utilizan con fines de conexión-interacción o investigativos (Barreto \& Jimenez, 2010; Curioso, Alvarado-Vásquez, \& Calderón-Anyosa, 2011; Dunlap \& Lowenthal, 2009; Forgiea, Duff, \& Ross, 2013; Fox \& Varadarajan, 2011). Tur y Marín (2015) evaluaron el uso de la plataforma Twitter para la realización de debates con estudiantes de educación en una universidad y analizaron el uso, la participación y la percepción de los estudiantes de la herramienta y concluyen que había incentivado la participación y ayudado a la comprensión. Si bien se ha utilizado la herramienta para interactuar, debatir e incluso evaluar, se puede decir que el uso de Twitter juego de roles puede considerarse una novedad.

Tomando en cuenta estos antecedentes, el estudio consideró las siguientes preguntas de investigación en relación a la experiencia pedagógica: 1) ¿Qué impacto tuvo el juego de rol "tuiterazo" en la comprensión del evento histórico de la independencia latinoamericana y de la posición de distintos actores en los estudiantes de B1?; ¿Qué niveles de comprensión desarrolló la mayoría de los estudiantes en relación a la posición de los actores? 2) ¿Qué impacto tuvo la práctica pedagógica del juego de rol "tuiterazo" en el desarrollo de la empatía en los estudiantes de B1? 3) ¿Qué nivel de satisfacción desarrolló la actividad en los estudiantes de B1 participantes?

\section{MARCO CONCEPTUAL}

Se entiende el constructivismo como paradigma teórico, pero también como enfoque de aprendizaje (Serrano \& Parra, 2011). Entre sus metodologías, se encuentra la EpC. El enfoque de enseñanza para la comprensión $(\mathrm{EpC})$ plantea que se demuestra la comprensión cuando el estudiante puede actuar en una realidad concreta y con un pensamiento flexible (Perkins, 1999). La EpC utiliza un marco de planificación que gira en torno al concepto de desempeños de aprendizaje. El docente diseña actividades que promueven la comprensión a partir del desarrollo de desempeños auténticos de aprendizaje (Ordóñez, 2004) relacionados a la vida diaria o al uso del conocimiento de una disciplina. La planificación considera 1) los contenidos que vale la pena aprender, que se denominan como tópicos generativos 2) los aspectos que deben ser comprendidos que se materializan en metas de comprensión 3) los desempeños que comprensión, que deben avanzar en niveles de complejidad y ser auténticos y 4) la evaluación diagnóstica continua (Perkins, 1999).

La EpC plantea la existencia de cuatro niveles de comprensión: comprensión ingenua, de principiante, de aprendiz y de maestría (Wiske, 1999) que describen diferentes niveles de logro y de desarrollo de capacidades del estudiante en relación a la flexibilidad con que usan y aplican los conceptos a situaciones reales, la profundidad de sus comprensiones, su posición crítica frente a dichos saberes y su autonomía en el desarrollo de desempeños.

La comprensión de la historia plantea algunas problemáticas, unas derivadas de su forma de enseñanza y otras relacionadas a su condición de ciencia social. La historia implica el desarrollo de un pensamiento abstracto complejo, ya que, a diferencia de las ciencias naturales, no puede reproducirse un fenómeno o evento histórico como se reproduce un fenómeno natural en un laboratorio (Prats, 2001). A éstas se suman complicaciones relacionadas a cómo se comprenden los conceptos de época, la multicausalidad detrás de los eventos y a la construcción del tiempo pasado (Asencio, Carretero, \& Pozo, 1989; Berrocoso, 2010; Comes \& Trepat, 2000; Prats, 2001; Zaragoza, 1989) que dificultan el desarrollo del denominado pensamiento histórico que implica que los estudiantes pasen de entender la historia como "evento" a entenderla como "relatos" producidos por los actores que deben ser analizados y evaluados (Bain, 2005). A este desafío, se le suma en América Latina, una dimensión política: la necesidad de desarrollar una lectura de la historia con una mirada poscolonial y que rescate a los actores subalternos (Mendieta, 1998; Prakash, 1997; Spivak, 2010).

El concepto de empatía se define de formas distintas (Cherubini, 2011; Fernández, 2011). Mientras hay autores que ven la empatía desde una dimensión racional, otros la ubican en planos emocionales (Fernández, 2011), sin embargo se la podría entender como un proceso y producto integral que incluye una respuesta afectiva consecuencia de procesos cognitivos (Feshbach, 1978). En esa misma línea, hay corrientes de pensamiento que 
no separan la razón de la emoción, y que consideran a la emoción como dominios de acción (Maturana, 1995) que influyen en la comprensión, relación e interacción humana con el mundo. Se presentan como componentes de la empatía la capacidad de compartir el pensamiento o sentimiento de otro y de adoptar su perspectiva subjetiva (Fernández, 2011).

En este caso del juego de rol, la empatía implica no solo la comprensión o adopción de un sentimiento (por ejemplo, la indignación, en el caso de actores históricos que se encuentran en una situación de abuso), sino de una racionalidad (por ejemplo, analizar las desventajas para las colonias latinomericanas de las Reformas Borbónicas). Es importante entender la empatía como la capacidad de adoptar tanto la perspectiva como el rol de otro, a partir de procesos de identificación y esto implica el desarrollo de orientaciones emocionales y cognitivas (Feshbach, 1978). Autores confirman la relación entre la empatía y comportamientos pro sociales (Roberts \& Strayer, 1996), por lo que es importante desarrollarla.

El aprendizaje basado en juegos es parte de los enfoques relacionados a la gamificación, que promueven el uso de juegos para trabajo o formación (Campos, 2012). En el mundo educativo, existe la distinción entre juegos divertidos y juegos serios, los serios son aquellos diseñados con objetivos de educación, entrenamiento e información (Michael y Chen, 2006 citado por Marcano (2008). En este caso, nos encontramos frente a un juego de roles. Esta técnica solicita a los participantes comportarse como si representaran personajes a partir de un tema imaginario (Saegesser, 1991; Urbina Criado, Salgado, \& Durán, 2010). Los juegos de roles permiten explorar cómo piensan otros (Brell, 2006) a partir de la interpretación de un personaje. Un juego de rol permitiría desarrollar la fantasía histórica (García \& Miranda, 2010). Los juegos de roles cumplen muchas funciones, entre ellas, el análisis de situaciones - como un evento histórico - desde diversos puntos de vista y el desarrollo de la empatía con otros grupos, como los actores sociales de la independencia. En el mundo educativo, se identifica que actividades como el juego de roles, los dilemas morales y las relacionadas a la identificación de los sentimientos de otros como contribuyentes al aprendizaje de la empatía (Fernández, 2011). Las simulaciones virtuales, entendidas como un juego de rol en entornos virtuales, permiten la comprensión de conceptos abstractos, como los históricos, a partir de la modificación de variables espacio-temporales (Berrocoso, 2010). García y Miranda (2010) plantean la importancia de los juegos de rol para la didáctica de historia y de proporcionar fuentes históricas a los actores para que puedan desempeñar su papel.

Los juegos pueden desarrollarse en el marco del aprendizaje invisible (Cobo Romaní \& Moravec, 2011), un enfoque que retoma el constructivismo y lo sitúa en el contexto de las nuevas tecnologías de la información y del conectivismo. Desde esta concepción, las tecnologías son invisibles, es decir, no se aprende "de" la tecnología, sino "con" la tecnología. Las TICS, cuando funcionan de forma integrada en el aprendizaje, promueven el compromiso activo, la participación grupal, la retroalimentación y las conexiones con contextos reales (Hernández Requena, 2008). La integración de las TICS se vuelve necesaria y consistente más aún en el trabajo con niños y adolescentes nativos digitales, que es como se denomina a la generación que nació y ha crecido con la tecnología (Prensky, 2011) y que es el caso de los estudiantes de educación media.

\section{METODOLOGÍA}

\section{Los tuiterazos en el contexto nacional}

En el ámbito nacional, la red social Twitter se ha convertido en un espacio de debate, opinión y visibilización de diferentes actores social, quienes se convocan a "tuiterazos". La convocatoria incluye la definición de un hashtag específico con el que se tuitea durante aproximadamente una hora para visibilizar opiniones en relación a dicha problemática. Es frecuente que los actores y movimientos sociales se citen en la red para participar en "tuiterazos" en horarios específicos alrededor de temas ambientales (por ejemplo \#Yasuni \#NoToquenElYasuni, 2013), protección animal (por ejemplo \#VidaParaAtena, 2015), desalojos (por ejemplo \#TrinitariaEnPaz, 2015), género (por ejemplo \#vergüenzajusticiaEC, 2014), entre otros. Estas convocatorias tienen como objetivo generar presencia en las redes sociales con el objetivo de estar en los trending topics de un tema, un ranking que se genera a partir de palabras o frases más repetidas en la red social Twitter.

\section{La actividad pedagógica}

La actividad pedagógica se inspiró en este tipo de convocatoria que utilizan los agentes sociales para diseñar un juego de rol que plantea esta situación hipotética: Los actores sociales relacionados a los procesos de independencia del SXIX en América Latina se convocan en Twitter para visibilizar sus posiciones en torno a dicho proceso. Consecuentes con la EpC, se diseñó la actividad²

a) el tópico generativo, eran los procesos de independencia en el SXIX;

\footnotetext{
${ }^{2}$ La investigadora participó en el diseño de la actividad como coordinadora del área de Sociales de la institución, además de las docentes de historia y de investigación.
} 
b) las metas de comprensión eran el análisis crítico de la independencia latinoamericana, considerando sus causas principales (Reformas Borbónicas), sus consecuencias y los actores principales involucrados (españoles, pensadores latinoamericanos, criollos e indígenas) con sus perspectivas del hecho;

c) el desempeño de comprensión diseñado se denominó “un tuiterazo por la independencia” que podría considerarse como auténtico, ya que los tuiterazos son convocatorias reales del mundo del activismo social contemporáneo.

La docente organizó desempeños preliminares, en los que se analizaban textos divulgativos sobre las causas y consecuencias de los procesos de independencia y la participación de actores. Los estudiantes fueron conformados en parejas o grupos de tres. A cada grupo se le asignó un rol en el juego: criollo, indígena, español, personaje histórico, pensador de la independencia. Cada grupo debía investigar la posición de su actor en relación a la independencia y, a partir de ella: 1) crear una cuenta-personaje ficticia que represente a su actor; 2) crear contenidos relacionados su posición. El diseño de cuenta debía incluir: 1) nombre 2) usuario 3) avatar (imagen del personaje o de algo de la época) 4) bio. A partir de los factores y causas de la independencia externas e internas y la posición de su actor, los estudiantes debían diseñar por lo menos diez tuits que realizaría ese personaje en el tuiterazo. Los tuits podían incluir imágenes o memes. El diseño debía ser expuesto oralmente acompañado de una justificación histórica. Se planteó el desempeño de forma integrada con la materia de computación y el departamento de sistemas, debido a que la docente de la materia tenía un escaso nivel de manejo de la tecnología y las redes sociales.

Como era importante promover una interacción “más auténtica” como sucede en los tuiterazos reales, se invitó a un grupo de Bachillerato 3 a participar en la actividad de forma secreta. Estos estudiantes ya conocían el tema y abrieron sus propias cuentas representando a los mismos actores sociales.

El día del tuiterazo, los estudiantes de cada curso se convocaron en el aula de computación, en la que se encontraban tanto la docente de Sociales, como la de computación. Cada tuiterazo duró aproximadamente cuarenta minutos. El timeline se proyectaba en una pantalla. La docente promovía procesos de interacción. Cada tuiterazo produjo entre 400 y 500 tuits, lo que implica un nivel de participación alto, si se considera lo que podría lograrse en un debate de carácter oral.

\section{Población}

La práctica pedagógica del juego de rol “tuiterazo" se realizó con los 3 grupos de Bachillerato 1 de la institución, es decir con el universo de estudiantes de ese nivel. El nivel cuenta con 76 estudiantes.

Se tomó datos del universo de estudiantes a partir de una encuesta. El estudio contó con 72 participantes (21 en el grupo 1, 26 en el grupo 2 y 25 en el grupo 3), que corresponden al 94,73\% del universo que incluye doce estudiantes con necesidades especiales con dificultades de lenguaje y comunicación que corresponden al $15,78 \%$ del total. Su nivel de competencia tecnológica relacionado al manejo de la red social era alto: la mayoría de estudiantes poseían una cuenta de Twitter $(68,06 \%)$ y consideraban que tenían un buen o muy buen manejo de ésta $(61,11 \%)$.

Este antecedente es relevante, porque los resultados y dinámica de la actividad hubiesen podido ser distintas si la red social era desconocida o sus niveles de uso y manejo hubiesen sido deficientes.

\section{Diseño de investigación}

Se realizó un diseño de investigación-acción de post- prueba con un enfoque cuantitativo y se estudió la respuesta del grupo en torno a las variables 1) comprensión del evento histórico 2) posición de distintos actores y 3) empatía 4) satisfacción (Cuadro 1).

\begin{tabular}{|l|l|l|}
\hline Variable & Operativización & Indicador \\
\hline $\begin{array}{l}\text { Comprensión del evento } \\
\text { histórico }\end{array}$ & $\begin{array}{l}\text { Comprensión de la naturaleza de los eventos } \\
\text { históricos como producto de diferentes perspecti- } \\
\text { vas de actores históricos }\end{array}$ & $\begin{array}{l}\text { Pregunta de opciones múltiples sobre actores que } \\
\text { participan en un evento histórico }\end{array}$ \\
\hline $\begin{array}{l}\text { Posición de distintos ac- } \\
\text { tores }\end{array}$ & $\begin{array}{l}\text { Capacidad de enunciación de los argumentos cen- } \\
\text { trales que resumen la posición de los actores }\end{array}$ & $\begin{array}{l}\text { Pregunta abierta sobre aprendizajes declarados } \\
\text { por parte de los estudiantes sobre las posiciones } \\
\text { de cada actor categorizados por temas }\end{array}$ \\
\hline Empatía & $\begin{array}{l}\text { Percepción de desarrollo de capacidad para pon- } \\
\text { erse en el rol del actor designado y de comprender } \\
\text { el punto de cita de otro }\end{array}$ & $\begin{array}{l}\text { Escalas para medir resultado de la actividad en tér- } \\
\text { minos de su capacidad para desarrollar empatía }\end{array}$ \\
\hline Satisfacción & $\begin{array}{l}\text { Apreciación general de la actividad } \\
\text { Escalas para medir resultado de la actividad en tér- } \\
\text { minos de su capacidad para desarrollar empatía }\end{array}$ \\
\hline
\end{tabular}

Cuadro 1. Operativización de variables.

Fuente: Elaboración propia a partir de las definiciones de autores del marco conceptual. 
Como herramienta de investigación se aplicó un cuestionario. El cuestionario fue de carácter anónimo, para que se pudiesen expresar en relación a la actividad con libertad y no lo sintieran como una forma de evaluación. Constaba de diferentes formatos de preguntas: 1) preguntas cerradas de opción múltiple; 2) preguntas abiertas sobre sus aprendizajes generales y sobre las posiciones de cada uno de los actores; 3) escalas de Likert con ítems relacionados a aprendizajes de historia o manejo de la tecnología, y escalas de valoración. El cuestionario tomó como base la herramienta propuesta por Durán Caneo (2013) para medir aprendizaje y satisfacción de una actividad que había sido validada y utilizaba el enfoque de Aprendizaje basado en Juegos virtuales a la que se le realizaron modificaciones. Desde el punto de vista ético, se contó con el consentimiento de la Dirección de la institución y de las docentes involucradas. La información se procesó en una base de datos y se realizó un análisis de frecuencia. En el caso de las preguntas abiertas, se revisaron y se agruparon las comprensiones a partir de categorías que se resumían en enunciados. A partir de las tendencias que se presentan en las comprensiones, se realizó una interpretación de las mayoritarias, tomando como referencia los niveles de comprensión propuestos por la EpC y los objetivos del módulo.

\section{RESULTADOS}

Los resultados cuantitativos se presentan organizados por variable en tablas de porcentajes. Los resultados se presentan ordenados de mayor a menor, salvo en el caso de las escalas, para evidenciar los niveles de aceptación.

\section{Comprensión del evento histórico y posición de actores involucrados}

En términos de las metas de comprensión, éstas se cumplieron. En la Tabla 1 se evidencia que el 76,39\% declara que la comprensión de un evento histórico implica conocer las diferentes perspectivas de diferentes actores involucrados, sin sobrestimar unos o descartar otros.

\begin{tabular}{|c|c|c|c|c|c|}
\hline $\begin{array}{l}\text { Después del ejercicio, usted diría que para entender los eventos históri- } \\
\text { cos, debe considerarse... }\end{array}$ & Grupo 1 & Grupo 2 & Grupo 3 & Total & $\%$ \\
\hline $\begin{array}{l}\text { el punto de vista de diferencias actores o grupos sociales que tienen difer- } \\
\text { entes visiones }\end{array}$ & 16 & 20 & 19 & 55 & 76,39 \\
\hline $\begin{array}{l}\text { el punto de vista de los actores principales, es decir, los vencedores, como } \\
\text { los criollos, líderes y pensadores latinoamericanos }\end{array}$ & 3 & 1 & 5 & 9 & 12,50 \\
\hline el punto de vista de los vencidos, en este caso, los españoles & 0 & 0 & 0 & 0 & 0,00 \\
\hline el punto de vista de las minorías, en este caso los indígenas & 2 & 3 & 1 & 6 & 8,33 \\
\hline no contesta & 0 & 2 & 0 & 2 & 2,78 \\
\hline Total & 21 & 26 & 25 & 72 & 100,00 \\
\hline
\end{tabular}

Tabla 1. Comprensión del evento histórico.

Fuente: Elaboración propia. Cuestionario aplicado a 72 estudiantes de B1.

Esto se constata además con los resultados de la pregunta abierta que invitaba a los estudiantes a declarar qué habían aprendido de la actividad y que se evidencian en la Tabla 2. La Tabla sistematiza las respuestas a pregunta abierta: “¿Qué considera que aprendió en historia de la actividad de "tuiterazo por la independencia?” Cada enunciado de los estudiantes se sistematizó en una categoría independiente, ya que algunos estudiantes declaraban varios aprendizajes, por lo que el número de respuestas de cada grupo es superior a la muestra original.

En la tabla se evidencia que el 38,89\% declara haber aprendido sobre los otros actores involucrados en el proceso de independencia. Se procesaron todos los enunciados realizados por los estudiantes en las preguntas abiertas.

\begin{tabular}{lccccc}
\hline & Grupo 1 & Grupo 2 & Grupo 3 & Total & $\%$ \\
\hline Aprendizajes generales declarados & & & & & \\
\hline Empatía & 1 & 2 & 4 & 7 & 7,78 \\
Aprender a persuadir a otros o defender una idea & 3 & 2 & 1 & 6 & 6,67 \\
Trabajo en equipo & 0 & 1 & 0 & 1 & 1,11 \\
Aprendizajes específicos declarados & & & & & \\
\hline Aprender de otros actores involucrados (ideas y posturas) & 11 & 13 & 11 & 35 & 38,89 \\
Conocer a los personajes históricos involucrados & 6 & 4 & 9 & 19 & 21,11 \\
Identificar situaciones de injusticia & 3 & 0 & 4 & 7 & 7,78 \\
Formar mi criterio sobre el evento & 3 & 2 & 0 & 5 & 5,56 \\
Proyectarse en otra época & 0 & 0 & 2 & 2 & 2,22 \\
Otro & 1 & 3 & 0 & 4 & 5,55 \\
Nada & 0 & 1 & 1 & 2 & 2,22 \\
No contesta & 0 & 1 & 0 & 1 & 1,11 \\
Total & & & & & 100,0 \\
\hline
\end{tabular}

Tabla 2. Aprendizajes de la actividad.

Fuente: Elaboración propia. Cuestionario aplicado a 72 estudiantes de B1. 
En relación a los argumentos y posiciones de los actores aprendidas, en la Tabla 3 los estudiantes demuestran en términos generales - comprensiones de las posiciones más importantes de los actores. La tabla sistematiza las respuestas a preguntas abiertas a partir de las que se construyeron categorías excluyentes. Las preguntas solicitaban: 1) Resuma los argumentos o posiciones sobre la independencia del grupo al que representaba en Twitter [coloque por lo menos 2 e indique por qué esa posición. Puede colocar ideas, acciones, posiciones, etc.]; 2) Resuma los argumentos o posiciones sobre la independencia de los OTROS grupos (indígenas, españoles, pensadores, criollos) que leyó en Twitter [por lo menos UNO de cada uno de esos grupos]. Cada idea de los estudiantes sobre los actores se sistematizó en una categoría independiente, ya que algunos estudiantes declaraban varias ideas en relación a la posición de los actores, por lo que el número de respuestas de cada grupo es superior a la muestra.

En la categoría "idea equivocada" se colocaron las respuestas de los estudiantes que evidenciaban errores de comprensión sobre el papel del actor. En el caso de los indígenas la categoría "reafirmación identitaria" corresponde a respuestas de los estudiantes que valoraban la cultura indígena. El caso de los españoles es interesante, ya que se presentaron tanto respuestas que agrupaban una "lectura colonial" en la que se coloca el "aporte" de estos a la cultura indígena o de su cultura como "superior" (posición que es válida en el contexto del juego de rol y que corresponde a la época), pero también lograban "lecturas poscoloniales" del evento, en las que los estudiantes realizaban un juicio crítico de la posición de los españoles en torno a su contribución a América y una comprensión de lo indígena como un actor poseedor de una cultura, una espiritualidad y una visión propia deslegitimada por la visión colonial.

\begin{tabular}{|c|c|c|c|c|c|}
\hline & Grupo 1 & Grupo 2 & Grupo 3 & Total & $\%$ \\
\hline \multicolumn{6}{|l|}{ Argumentos de otros actores Indígenas } \\
\hline Situación de abuso (esclavitud, impuestos) & 4 & 9 & 6 & 19 & 35,85 \\
\hline Reafirmación identitaria o conciencia histórica & 2 & 5 & 7 & 14 & 26,42 \\
\hline Necesidad de libertad & 4 & 1 & 4 & 9 & 16,98 \\
\hline Solicitud de igualdad con criollos & 1 & 2 & 0 & 3 & 5,66 \\
\hline Idea equivocada & 0 & 0 & 2 & 2 & 3,77 \\
\hline Otro & 0 & 4 & 2 & 6 & 11,32 \\
\hline Total & & & & 53 & 100,0 \\
\hline \multicolumn{6}{|l|}{ Pensadores } \\
\hline Libertad & 4 & 4 & 4 & 12 & 37,50 \\
\hline Idea equivocada & 0 & 2 & 5 & 7 & 21,88 \\
\hline Desconcentración o igualdad de poder & 0 & 0 & 3 & 3 & 9,38 \\
\hline Conciencia de patria o de nacionalismo & 1 & 0 & 0 & 1 & 3,13 \\
\hline Otro & 2 & 4 & 3 & 9 & 28,13 \\
\hline Total & & & & 32 & 100,0 \\
\hline \multicolumn{6}{|l|}{ Españoles } \\
\hline Lectura colonial "cultura, religión, educación" & 9 & 8 & 14 & 31 & 49,21 \\
\hline Impulso a desarrollo (visión paternalista) & 5 & 3 & 8 & 16 & 25,40 \\
\hline Referencia a reformas borbónicas & 0 & 0 & 3 & 3 & 4,76 \\
\hline Lectura poscolonial "cultura, religión, educación" & 1 & 0 & 1 & 2 & 3,17 \\
\hline Otras dimensiones de la conquista & 1 & 0 & 0 & 1 & 1,59 \\
\hline Idea equivocada & 0 & 1 & 0 & 1 & 1,59 \\
\hline Otro & 4 & 3 & 2 & 9 & 14,29 \\
\hline Total & & & & 63 & 100,00 \\
\hline \multicolumn{6}{|l|}{ Criollos } \\
\hline Ocupar cargos políticos & 2 & 10 & 5 & 17 & 43,59 \\
\hline Igualdad (abuso) & 2 & 3 & 1 & 6 & 15,38 \\
\hline Libertad & 0 & 1 & 2 & 3 & 7,69 \\
\hline Idea equivocada & 0 & 0 & 3 & 3 & 7,69 \\
\hline Solicitud de descentralización del poder & 2 & 0 & 0 & 2 & 5,13 \\
\hline Otro & 2 & 2 & 4 & 8 & 20,51 \\
\hline Total & & & & 39 & 100,0 \\
\hline
\end{tabular}

Tabla 3. Posiciones de los actores de la independencia enunciadas.

Fuente: Elaboración propia. Cuestionario aplicado a 72 estudiantes de B1.

Se puede analizar estos aprendizajes considerando los niveles de aprendizaje del EpC y se concluye que estos tienen distintos niveles. En el caso de los actores indígenas, podría decirse que los estudiantes alcanzan comprensiones de maestría, ya que un grupo importante $(38,85 \%)$ identifica que durante el proceso de independencia este grupo social se encontraba en una situación de esclavitud y abuso que en realidad no cambió con la independencia. Es interesante que se presenten respuestas con posiciones poscoloniales en las que se evidencia una conciencia histórica de la invasión y explotación de los países europeos. Sobre los pensadores, en cambio podríamos decir que se presentan comprensiones ingenuas: los aprendizajes enunciados por los estudiantes están relacionados a la libertad en general sin mayores precisiones sobre por qué obtenerla (37,50\%) o su relación con el pensamiento ilustrado y además se suma una presencia considerable de ideas equivocadas sobre este grupo (21,88\%), por ejemplo, que estaban a favor de los indígenas. Por lo tanto, el conocimiento de ese actor debe reforzarse. En el caso de los españoles, podríamos decir que se presentan comprensiones de aprendiz, ya que si bien se hacen lecturas coloniales válidas para el juego de rol (49,21\%), los estudiantes debieron enunciar las Reformas Borbónicas como necesarias desde la perspectiva desde actor que 
eran uno de los aspectos centrales de la política española de la época y un factor importante para desencadenar la independencia. En el caso de los criollos en cambio, se presentan comprensiones de nivel de maestría, ya que casi la mayoría señala la solicitud de poder ocupar cargos públicos $(43,59 \%)$ que es lo que demandaban estos actores en la época. Si analizamos los porcentajes generales de errores de comprensión, una minoría entre el $1 \%$ y $21 \%$ - cometió errores, por lo que la mayoría comprendió las posiciones centrales de los actores.

\section{Empatía}

Los estudiantes perciben que desarrollaron altos niveles de empatía que se evidencian en las respuestas a la escala de valoración que se presenta en la Tabla 4. Sobre el tema de la empatía, se evidencia que la mayoría $(62,50 \%)$ declaró que estaba muy de acuerdo en que la actividad les ayudó a ponerse en el lugar del grupo o actor que representaba y entender sus ideas, motivos, posiciones y sentimientos. Casi la mitad estaba bastante de acuerdo $(48,61 \%)$ o muy de acuerdo $(40,28 \%)$ en que la actividad les ayudó a entender las ideas, motivos, posiciones y sentimientos de los demás grupos o actores de la independencia. Finalmente, casi la mitad $(48,61 \%)$ estaba muy de acuerdo y casi la tercera parte (26,39\%) estaba bastante de acuerdo en que la actividad les había ayudado a contrastar el punto de vista de su actor-personaje con el de otros actores.

\begin{tabular}{|c|c|c|c|c|c|c|}
\hline \multicolumn{2}{|l|}{ Argumentos o posiciones de otros actores } & G1 & G2 & G3 & Total & $\%$ \\
\hline \multirow{6}{*}{$\begin{array}{l}\text { Me ayudó a ponerme en el lugar del grupo } \\
\text { o actor que representaba y entender sus } \\
\text { ideas, motivos, posiciones y sentimientos }\end{array}$} & Muy de acuerdo & 15 & 16 & 14 & 45 & 62,50 \\
\hline & Bastante de acuerdo & 5 & 7 & 7 & 19 & 26,39 \\
\hline & Ni de acuerdo ni en desacuerdo & 1 & 2 & 4 & 7 & 9,72 \\
\hline & Un poco en desacuerdo & 0 & 0 & 0 & 0 & 0,00 \\
\hline & Totalmente en desacuerdo & 0 & 0 & 0 & 0 & 0,00 \\
\hline & No contesta & 0 & 1 & 0 & 1 & 1,39 \\
\hline \multirow{6}{*}{$\begin{array}{l}\text { Me ayudó a entender las ideas, motivos, } \\
\text { posiciones y sentimientos de los demás } \\
\text { grupos o actores de la independencia }\end{array}$} & Muy de acuerdo & 6 & 11 & 12 & 29 & 40,28 \\
\hline & Bastante de acuerdo & 13 & 10 & 12 & 35 & 48,61 \\
\hline & Ni de acuerdo ni en desacuerdo & 2 & 3 & 1 & 6 & 8,33 \\
\hline & Un poco en desacuerdo & 0 & 0 & 0 & 0 & 0,00 \\
\hline & Totalmente en desacuerdo & 0 & 1 & 0 & 1 & 1,39 \\
\hline & No contesta & 0 & 1 & 0 & 1 & 1,39 \\
\hline \multirow{6}{*}{$\begin{array}{l}\text { Me ayudó a contrastar el punto de vista } \\
\text { de mi actor-personaje con el de otros } \\
\text { actores }\end{array}$} & Muy de acuerdo & 9 & 13 & 13 & 35 & 48,61 \\
\hline & Bastante de acuerdo & 8 & 5 & 6 & 19 & 26,39 \\
\hline & Ni de acuerdo ni en desacuerdo & 2 & 5 & 4 & 11 & 15,28 \\
\hline & Un poco en desacuerdo & 2 & 1 & 2 & 5 & 9,64 \\
\hline & Totalmente en desacuerdo & 0 & 1 & 0 & 1 & 1,39 \\
\hline & No contesta & 0 & 1 & 0 & 1 & 1,39 \\
\hline Total & & 21 & 26 & 25 & 72 & 100,0 \\
\hline
\end{tabular}

Tabla 4. Aprendizajes obtenidos en el juego de roles.

Fuente: Elaboración propia. Cuestionario aplicado a 72 estudiantes de B1.

En términos generales la satisfacción es alta y se evidencia en la Tabla 5: la mayoría está muy de acuerdo $(56,94 \%)$ y bastante de acuerdo $(23,61 \%)$ en que Twitter fue apropiado para motivar el aprendizaje; está muy de acuerdo $(55,56 \%)$ y bastante de acuerdo $(33,33 \%)$ en que la actividad fue apropiada para practicar el material estudiado y está muy de acuerdo $(68,06 \%)$ y bastante de acuerdo $(23,61 \%)$ en que la actividad fue divertida. Incluso el 90,28\% indicó que recomendaría esta actividad a otros compañeros y en una pregunta abierta por sugerencias, el 25,93\% (que era la categoría más alta) sugirió extender el tiempo de la actividad, lo que podría interpretarse como un indicador de un significativo involucramiento en ésta.

\begin{tabular}{|c|c|c|c|c|c|c|}
\hline & & G1 & G2 & G3 & Total & $\%$ \\
\hline \multirow{4}{*}{$\begin{array}{l}\text { La red Twitter fue apropiada para motivar } \\
\text { el aprendizaje }\end{array}$} & Muy de acuerdo & 12 & 14 & 15 & 41 & 56,94 \\
\hline & Ni de acuerdo ni en desacuerdo & 5 & 4 & 4 & 13 & 18,06 \\
\hline & Totalmente en desacuerdo & 0 & 0 & 0 & 0 & 0,00 \\
\hline & No contesta & 0 & 1 & 0 & 1 & 1,39 \\
\hline \multirow{4}{*}{$\begin{array}{l}\text { La actividad fue apropiada para practicar } \\
\text { el material estudiado }\end{array}$} & Muy de acuerdo & 13 & 13 & 14 & 40 & 55,56 \\
\hline & Un poco en desacuerdo & 0 & 0 & 0 & 0 & 0,00 \\
\hline & Totalmente en desacuerdo & 0 & 1 & 0 & 1 & 1,39 \\
\hline & No contesta & 0 & 1 & 0 & 1 & 1,39 \\
\hline \multirow{2}{*}{ La actividad fue divertida } & Muy de acuerdo & 13 & 19 & 17 & 49 & 68,06 \\
\hline & Bastante de acuerdo & 6 & 5 & 6 & 17 & 23,61 \\
\hline
\end{tabular}

Tabla 5. Satisfacción.

Fuente: Elaboración propia. Cuestionario aplicado a 72 estudiantes de B1. 


\section{CONCLUSIONES}

El juego de rol "tuiterazo" logró desarrollar las metas de comprensión relacionadas a la unidad. La meta de comprensión principal de comprender los eventos históricos desde la visión de distintos actores se cumple. Los estudiantes identifican las posiciones centrales de todos los actores relacionados y una minoría presenta comprensiones equivocadas. Sin embargo, los niveles de comprensión de las posiciones de los actores presentan diferentes niveles, lo que implica trabajar en desempeños preliminares con mayor profundidad. El juego de rol también cumplió el objetivo de desarrollar empatía, tanto con el rol que se desempeñaba, como con las posiciones de los otros actores.

Como condiciones para el adecuado desarrollo del desempeño y de los logros de aprendizaje encontramos la realización de desempeños preliminares, que implican la investigación previa de la posición de los actores y el desarrollo de contenidos a tuitear. Esto significa entregar a los grupos fuentes más específicas relacionadas a cada actor y no fuentes sobre la independencia en general.

Desde la docencia, es importante considerar el nivel de manejo y uso de la tecnología y redes sociales que tiene el docente, porque docentes con escaso nivel en su manejo, pueden frustrase y una mala experiencia de integración de la tecnología, puede afectar incluso una buena actitud hacia ésta en el futuro. La incorporación del área de computación y de sistemas para brindar el apoyo en el desarrollo de competencias tecnológicas de los estudiantes y resolver problemas técnicos, son importantes para el adecuado desarrollo del desempeño. Es importante ubicar otro grupo que participe, sea del mismo curso o uno superior, porque aumenta la interactividad y la motivación.

\section{RECOMENDACIONES}

\section{Sobre el diseño de la actividad}

Como recomendaciones de carácter conceptual y político, el desarrollo de una lectura poscolonial de la historia, necesita identificar como personajes históricos también a los indígenas. Para profundizar la comprensión de la posición de estos actores y visibilizarlos más como actores políticos, se sugiere investigar las rebeliones indígenas y los nombres de personajes históricos indígenas específicos que se rebelaron previa o durante la independencia. Como crítica a este juego de rol, solo los actores "blancos" o "mestizos" tenían nombres y ninguna de las cuentas que crearon los estudiantes que representaban a los indígenas rescataba el nombre de un indígena en particular. Otros actores subalternos que podría visibilizar el ejercicio son las mujeres y los esclavos negros, que tuvieron participaciones y posiciones específicas.

Otra cuestión para mejorar este juego de rol, sería identificar eventos específicos previos y posteriores para ser nombrados en los contenidos de los tuits. Además, desde el punto de vista de los contenidos visuales, se podría utilizar la clase de computación para la busca de imágenes de la época o alusivas a ésta.

\section{Futuras investigaciones}

Una investigación futura podría considerar un diseño de tipo experimental, que utilice pre y post test de comprensiones; además de a realización de un análisis de contenido de los tuits producidos considerando los niveles de aprendizaje propuestos por la EpC y de entrevistas a los estudiantes sobre sus comprensiones tomando como objeto de estudio la participación.

El tuiterazo acerca a los estudiantes a un uso de redes sociales de carácter educativo y desarrolla la conciencia de participación política en temas sociales y políticos del presente. Este tipo de ejercicio - utilizando incluso celulares y dispositivos personales - podría aplicarse con facilidad en el debate de temas locales, nacionales o internacionales a partir de la identificación de hashtags específicos.

De la experiencia se puede concluir que la exploración de las posibilidades que presenta la red social Twitter para desarrollar debates o juegos de roles es un reto en el contexto del aprendizaje invisible. 


\section{REFERENCIAS}

Asencio, M. I. K. E. L., Carretero, M., \& Pozo, J. L. (1989). La enseñanza de las ciencias sociales. Madrid: Visor.

Bain, R. B. (2005). Cómo aprenden los estudiantes: historia en el aula de clase. Descargado de http://www.eduteka.org/ ComoAprendenLosEstudiantes.php

Barreto, C. R., \& Jimenez, A. C. (2010, Diciembre). El uso de facebook y twitter en educación. Lumen-Instituto de Estudios en Educación-IESE(11), 1-9. Descargado de https://guayacan.uninorte.edu.co/divisiones/iese/lumen/ ediciones/11/articulos/el-uso-de-facebook-y-twitter-en -educacion.pdf

Berrocoso, J. V. (2010). Aprendizaje de la historia y simulación. Tejuelo: Didáctica de la Lengua y la Literatura. Educación(9), 83-89. Descargado de http://dialnet.unirioja.es/ servlet/articulo?codigo $=3719337$

Campos, A. O. (2012, Diciembre). Crítica y conciliación de la ludificación. Descargado de http://maledictus.com.mx/ pedagogia/Ludificacion_AldoOjeda_1212.pdf

Cherubini, E. (2011). La función de la EMPATÍA en la educación. En Vii encuentro interdisciplinario de ciencias sociales y humanas. Argentina: Universidad Nacional de Córdoba. Descargado de http://publicaciones.ffyh.unc.edu.ar/ index.php/7encuentro/article/view/486

Cobo Romaní, C., \& Moravec, J. W. (2011). Aprendizaje invisible: Hacia una nueva ecología de la educación (Vol. 3). Barcelona: Edicions Universitat.

Comes, P., \& Trepat, C. (2000). El tiempo y el espacio en la didáctica de las ciencias sociales. Barcelona: Graò.

Curioso, W. H., Alvarado-Vásquez, E., \& Calderón-Anyosa, R. (2011, Mar.). Usando twitter para promover la educación continua y la investigación en salud en el Perú. Revista Peruana de Medicina Experimental y Salud Pública, 28(1), 163-164. Descargado de http://www.scielo.org.pe/scielo.php?pid= S1726-46342011000100031\&script=sci_arttext\&tlng=en

De Haro, J. J. (2010). Redes sociales para la educación. Descargado de http://www.cepazahar.org/ recursos/pluginfile.php/6425/mod_resource/content/

0/redes_sociales_educacion.pdf

Dunlap, J. C., \& Lowenthal, P. R. (2009). Tweeting the night away: Using twitter to enhance social presence. Journal of Information Systems Education, 20(2), 129-135. Descargado de http://jise.org/Volume20/20-2/Pdf/V20N2P129-abs.pdf

Durán Caneo, L. (2013). Aprendizaje basado en juegos como estrategia para el desarrollo de competencias específicas de educación (Trabajo final para la obtención del Título de Magíster en Educación Superior, Investigación e Innovaciones). Universidad Casa Grande, Ecuador.

Fernández, M. F. (2011). La empatía desde dos miradas: la evolución y la educación. En Vii encuentro interdisciplinario de ciencias sociales y humanas. Argentina: Universidad Nacional de Córdoba. Descargado de http://publicaciones.ffyh.unc .edu.ar/index.php/7encuentro/article/view/487

Feshbach, N. D. (1978). Studies of empathic behavior in children. En B. A. Maher (Ed.), Progress in experimental personality research (8a ed.). New York: Academic Press.

Forgiea, S. E., Duff, J. P., \& Ross, S. (2013). Twelve tips for using twitter as a learning tool in medical education. Medical Teacher, 35(1), 8-14. doi: 10.3109/0142159X.2012.746448

Fox, B. I., \& Varadarajan, R. (2011). Technology in pharmacy education: Use of twitter to encourage interaction in a multi- campus pharmacy management course. American journal of pharmaceutical education, 75(5). Descargado de http:// archive.ajpe.org/aj7505/aj750588/aj750588.pdf

García, J. R. C., \& Miranda, I. P. (2010). Fuentes históricas de los juegos de rol: un experimento para la didáctica de la historia antigua. Education in the Knowledge Society, 11(3), 149-167. Descargado de http://rca.usal.es/index.php/ revistatesi/article/view/7454

Grosseck, G., \& Holotescu, C. (2008, April). Can we use twitter for educational activities? En 4th international scientific conference, elearning and software for education. Bucharest, Romania: "Carol I" National Defense University. Descargado de https://adlunap.ro/else/papers/015.-697 .1.Grosseck\%20Gabriela-Can\%20we\%20use.pdf

Hernández Requena, S. R. (2008). El modelo constructivista con las nuevas tecnologías, aplicado en el proceso de aprendizaje. RUSC. Universities and Knowledge Society Journal, 5(2). doi: 10.7238/rusc.v5i2.335

Marcano, B. (2008, Noviembre). Juegos serios y entrenamiento en la sociedad digital. Teoría de la Educación: Educación y Cultura en la Sociedad de la Información, 9(3), 93107. Descargado de http://campus.usal.es/ teoriaeducacion/ rev_numero_09_03/n9_03_marcano.pdf

Maturana, H. (1995). Emociones y lenguaje en educación y política (8a ed.). Santiago de Chile: Dolmen.

Mendieta, E. (1998). Modernidad, posmodernidad y poscolonialidad: una búsqueda esperanzadora del tiempo. En S. Castro-Gómez \& E. Mendieta (Eds.), Teorías sin disciplina (latinoamericanismo, poscolonialidad y globalización en debate) (pp. 147-168). México: Miguel Ángel Porrúa.

Ordóñez, C. L. (2004, Diciembre). Pensar pedagógicamente desde el constructivismo. Revista de Estudios Sociales(19), 7-12. Descargado de http://res.uniandes.edu.co/view.php/ 401/index.php?id=401

Perkins, D. (1999). ¿qué es la comprensión? En M. S. Wiske (Ed.), La enseñanza para la comprensión: Vinculación entre la investigación y la práctica. Buenos Aires, Argentina: Paidós.

Prakash, G. (1997). Los estudios de la subalternidad como crítica post-colonial. En S. Rivera Cusicanqui \& R. Barragán (Eds.), Debates post coloniales: Unaintroducción a los estudios de la subalternidad (pp. 293-313). La Paz: SEPHIS, Editorial historias y Ediciones Aruwiry.

Prats, J. (2001). Enseñar historia: Notas para una didáctica renovadora. Mérida: Junta de Extremadura.

Prensky, M. (2011). Enseñar a nativos digitales. Madrid: SM.

Roberts, W., \& Strayer, J. (1996, April). Empathy, emotional expressiveness, and prosocial behavior. Child development, 67(2), 449-470. doi: 10.1111/j.1467-8624.1996.tb01745.x

Saegesser, F. (1991). Los juegos de simulación en la escuela. Madrid: Visor.

Serrano, J. M., \& Parra, R. M. P. (2011). El constructivismo hoy: enfoques constructivistas en educación. Revista Electrónica de Investigación Educativa, 13(1). Descargado de http://redie.uabc.mx/redie/article/view/268

Spivak, G. C. (2010). Crítica de la razón poscolonial: Hacia una crítica del presente evanescente. Madrid: AKAL.

Tur, G., \& Marín, V. I. (2015). Enriqueciendo el aprendizaje con social media: las percepcio-nes del alumnado sobre twitter usado en una actividad de debate. New Approaches in Educational Research, 4(1), 51-59. doi: 10.7821/naer.2015.1.102

Urbina Criado, M. O., Salgado, S. M., \& Durán, C. D. L. C. 
(2010). Herramientas para el aprendizaje colaborativo: una aplicación práctica del juego de rol. Education in the Knowledge Society, 11(3), 277-301. Descargado de http://rca.usal .es/index.php/revistatesi/article/view/7463

Wiske, M. S. (1999). Enseñanza para la comprensión: Vinculación entre la investigación y la práctica. Buenos Aires: Paidós.

Zaragoza, G. (1989). La investigación y la formación del pensamiento histórico del adolescente. En M. Carretero, J. I. Pozo, \& M. Asensio (Eds.), La enseñanza de las ciencias sociales. Madrid: Visor.

Cómo citar este artículo (APA):

Zerega-Garaycoa, M. M. (2015). Un “tuiterazo" por la independencia: una experiencia del uso de Twitter para la enseñanza de Historia. AtoZ: novas práticas em informação e conhecimento, 4(2), 74 - 83. Descargado de: http://dx.doi.org/10.5380/atoz.v4i2.43596 